\title{
Impact on adult children caring for elderly parents with a long-standing mental illness
}

\author{
Kuruvilla George*, Linda Fuller, Jennifer Ancell \\ Clinical Director, Aged Person's Mental Health, Eastern Health, Victoria, Australia \\ Senior Social Worker Aged Person's Mental Health, Eastern Health, Victoria, Australia \\ Senior Social Worker, Aged Person's Mental Health, Eastern Health, Victoria, Australia \\ *Corresponding author E-mail: kuruvilla.george@easternhealth.org.au
}

\begin{abstract}
Introduction: This research contrasts the experiences of long-term carers of elderly parents who have had a longstanding mental illness and first-time carers of parents who have become mentally unwell in old age. It was hypothesized that the children of elderly parents with a long-standing mental illness have differing needs when faced with the caring role compared to first-time carers of elderly mentally ill patients.

Method: Using a qualitative research methodology and interpretive perspective, eight carers were interviewed using a standardized questionnaire from which salient issues were drawn and analyzed.

Results: Long-term carers faced issues including a longer duration of care-giver stress, early entry into adult responsibilities of caring, frustrations concerning lack of recognition from mental health services and cumulative stress affecting long-term carers' significant relationships and health. First-time carers sought earlier access to mental health services, specialist information about mental health issues and coping strategies for the care-giving role.

Conclusion: It was found that both sets of carers (long-term and first-time), irrespective of the duration of their caring experiences, shared complex and competing demands on their physical and emotional resources. All carers were able to identify ways to improve support to improve support to children caring for ageing mentally ill parents.
\end{abstract}

Keywords::carers, impact, mental illness, elderly parents, adult children.

\section{Introduction}

Past research has identified issues faced by carers concluding that they are subject to overall poorer physical and psychological health.[1, 2, 3, 4,5] This is the case even where carers report positively on the experience of caring. [1,4] An expanding body of literature now also documents the difficulties encountered by children growing up with parents who are mentally ill, citing poor physical and psychological outcomes for children as they move into adulthood. $[6,7,8,9]$ There is however a paucity of research examining the experiences of adult children who care for an elderly parent with a psychiatric illness, in spite of the numbers of children engaged in parental care.

With better treatment and support, people with mental illness are living longer. By 2041 it is estimated that 500,000 people will be suffering dementia. As a result, significant numbers of children will be growing up with a parent who has mental illness and adult children will be called on to provide care for parents who will develop a mental illness in later life. It is essential that research be undertaken to examine the longitudinal impacts on children living with parents who have a mental illness, as well as the needs of adult children who are called on to support their parents for the first time in later life.

\section{Method and results}

With this in mind we made contact with 8 adult carers of elderly clients who were being case managed by our aged persons mental health service. A questionnaire formed the basis of a semi-structured interview designed to explore the impact and experiences of carers. It also asked about carer's perceived needs and suggestions for models of service delivery. Four carers were aged 40-50 years and four were over 50 caring for parents aged between 76 and 89 years. Only 1 carer was male, 5 were married, 2 were divorced and 1 single. Three of the carers were the 'only' child, 1 carer 
was a daughter-in-law and 4 carers had four or more siblings. Three of the carers were in full-time employment, 3 were not employed and 2 were employed part-time. None of the carers received any carer-related entitlements. Three of the clients were divorced, 3 were widowed and 2 were married, 2 were diagnosed with mental illness before the age of 18 , two between ages of 40-55, one between 65-75 and three after the age of 75. Three of the carer's were under 11 years when their parent became unwell, two were in their 20's, two in their 40's and one was over 50. Only one carer reported good knowledge of mental illness and having been given adequate information about their parent's illness at the time of diagnosis.

Carers all reported that caring for a parent had a direct bearing on their own mental health and all described other significant impacts in terms of family and relationships, social connectedness, self-esteem, personal security and employment or career aspirations. The long-term carers described physical, emotional or psychological stresses that they related directly to their caring roles with a parent with mental illness. One carer reported feeling unable to cope and four carers had sought counselling. Strategies like limit-setting, yoga, meditation, exercise, development of positive social networks and spirituality were used as means of reducing the burden of caring.

Overall there were more similarities than differences between the needs and experiences of the carers. A high incidence of "loyalty" was expressed as a motivator for caring for a parent, with six carers identifying this as a motivator followed by determinants of "love"[5] and "choice"[5]. At the same time, six carers reported that their relationships with their spouse and siblings had been negatively impacted by care-related responsibilities. Interpersonal distress occurred as a result of competing pressures between care-giving and family demands. Tensions resulting from the difficult behaviour of an unwell parent and the lack of appreciation for the caring role led to depression, guilt, anger, personal distress and feeling of manipulation and not being appreciated. In general the long-term caring role (compared to short-term carers) was perceived as a much more demanding role due to the length of years of caring and the constant emotional strain.

The need for timely diagnosis, information and counselling about mental illness was suggested at the time a parent became unwell, to be followed by mentoring, community support and courses about coping in the longer term. More than half of carers preferred programs concentrating on skills based workshops such as stress management, coping with difficult behaviour, medication and its side-effects, issues for grandchildren and future planning directives. Some carers did not have the emotional resources to deal with long, drawn out therapeutic endeavours but preferred pertinent information, reinforcing the idea that information empowers and brings a sense of personal mastery over stressful situations. [10] Six carers wanted to have direct contact with the treatment teams in the form of family meetings. Five carers believed there was a need for individual counselling from a health professional at a time of personal need. At other times there may be a need for tailored family interventions or group therapy where participants can be heard, validated and understood. The therapeutic gain from verbalising sentiments is well known and utilising support groups offered by mental health services is another positive alternative.

\section{Discussion}

This qualitative research project relied upon extensive narrative feedback from a small group of individuals engaged in the care of mentally ill parents. What emerges is that adult child carers, irrespective of the duration of their caring experiences, have complex and stressful lives, and spend a good deal of their time trying to negotiate competing demands on their emotional and physical resources. They largely do this with minimal help from wider formal support networks. As adult carers for a parent, many of the experiences of day to day activities overlapped, but were often contrasted by the duration and intensity of the experience of caring. Long-term carers were able to reflect on their early experiences and in what ways these impacted on their current lives. All carers in the study reported dilemmas that reflect the chronic, fluctuant nature of mental illness itself. All carers described stresses arising from undertaking ongoing supervising, monitoring and decision-making roles, and from witnessing parents' distress (due to illness, selfinjury or treatments). All carers presented as being well organised, vigilant and available to their parents. The majority had limited formal support in the caring role and had to juggle the competing demands of their family relationships or responsibilities.

Partners and children were often the primary support to carers, although many reported there to be ambivalence from these sources as well. Issues like being physically or emotionally exhausted and unwell emerged consistently as did elements of frustration about negotiating an often complex and sometimes even hostile health system.

In research by Veltman, Cameron and Steward [4], caregivers felt "let down" by the mental health system feeling that they found it "adversarial" rather than one of "advocacy", often reinforcing the negative view of caring and failing to express support for the positive aspects of caring. Such sentiments were echoed by the carers in the present study. Most keenly expressed throughout the research was the call by carers to be "believed" and "listened to". This sentiment was regarded not so much about their needs as carers, but was more about being treated respectfully and as competent people asserting their concerns about their parent's needs. Even as a small sample, their voices were loud and often unison in expressing the difficulties and joys of being a carer of a parent with mental illness. Detecting and 
appropriately responding to carer's vulnerabilities and strengths in a sensitive manner remains an essential priority for mental health practitioners and mental health services

\section{References}

[1] Heru A M. (2000) 'Family functioning, burden and reward in the care giving for chronic mental illness. Family, Systems and Health, Vol.18, No. 1

[2] Wilson I. (2001) 'The impact of mental illness on family members'. Australian Family Physician, Vol. 30 , No.9

[3] Spruytte N, Van Audenhove C, Lammertyn F, Storms G. (2002) 'The quality of the care-giving relationship in informal care for older adults with dementia and chronic psychiatric patients'. Psychology and Psychotherapy, Leicester, Vol. 75, Part 3, 295-312

[4] Veltman A, Cameron J I, Stewart D E. (2002) 'The experience of providing care to relatives with chronic mental illness'. Journal of Nervous and Mental Disease, Vol. 190, No 2

[5] Bhatia S, Tucker V, Kapoor G. (2003) 'Assessment of burden experienced by caregivers of patients suffering from schizophrenia'. Journal of Personality and Clinical Studies, Vol. 19 (10), 77-88

[6] Reder P, McClure M, Jolley A. (2000) 'Interfaces between child and adult mental health'. In Family matters: interfaces between child and adult mental health, Routledge 2000

[7] Benjet C, Azar S T, Kuersten-Hogan R. (2003) 'evaluating the parental fitness of psychiatrically diagnosed individuals: advocating a functional-contextual analysis of parenting'. Journal of Family Psychology, Vol 17, No.2, 238-251

[8] Mordoch E, Hall W A. (2002) 'Children living with a parent who has a mental illness: A critical analysis of the literature and research implications'. Archives of psychiatric Nursing, Vol. XVI, No.5, 208-216

[9] Bijil R V, Cuijpers P, Smit F. (2002) 'Psychiatric disorders in adult children of parents with a history of psychopathology'. Social Psychiatry and Psychiatric Epidemiology, 37, 7-12

[10] Brooks R B. (1994) 'Children at risk: Fostering resilience and hope'. American Orthopsychiatry Association, 64 (4), $545-553$ 